\title{
Electromagnetic damper using brushless direct drive DC motor
}

\author{
Taichi MATSUOKA* \\ *School of Science and Technology, Meiji University \\ 1-1-1 Higashimita, Tama-ku, Kawasaki-shi, Kanagawa 214-8571, Japan \\ E-mail: matsuoka@meiji.ac.jp
}

Received: 28 June 2018; Revised: 25 September 2018; Accepted: 23 October 2018

\begin{abstract}
To fabricate a semiactive type of damper that is comparatively compact and has low mechanical loss, the author developed an electromagnetic damper using a brushless direct drive DC motor. The damper comprises a ball screw mechanism and a brushless direct drive DC motor that acts as a generator. The motor consists of a rotor and a three-phase stator, and it is installed at the tip of the screw shaft. The lack of contact between the rotor and stator is an advantage. When a linear motion is converted into a rotational motion by the ball screw mechanism, the rotor is rotated, and electrical driving power is generated by electromagnetic induction. The damping effect of each phase is caused by kinetic energy dissipation by the electrical resistances connected to each terminal of coils. In this study, a test damper is fabricated, and theories of resisting force and rise time are introduced. Performance tests are carried out to confirm the theories and the dynamic properties of the damper for two winding configurations, and the experimental results are compared with the theoretical results. The damping effect is greatly generated and smoothly, but saturated in high frequency range, and switched quickly by SSR. It is possible to make the damper more efficient, compact, and sensitive. Finally, theories and the dynamic properties of the damper are confirmed.
\end{abstract}

Keywords : Damper, Vibration control device, Damping, Vibration control, Direct drive motor, Brushless

\section{Introduction}

In recent years, vibration control devices such as semiactive-type dampers have been used widely in structures, vehicles, trains, and machines to achieve a more efficient vibration reduction. In general, passive-type dampers are simple and stable, whereas active-type dampers are complex, high-performance, and expensive. Semiactive-type dampers have advantages in terms of control, and they remain stable under malfunction. Many types of semiactive dampers have been developed by researchers, for example, oil dampers, friction dampers, and functional fluid dampers. Here the author focuses on electromagnetic dampers. Most electromagnetic dampers comprise a motor that acts as a generator, and the damping effect is generated by the dissipation of electrical energy. When the motor condition is electrically switched by signals, the damper characteristics can be controlled. In the past, mechatronic dampers (Chiba and Kobayashi, 1984, Sunakoda, et al., 1995) and regenerating dampers, which have a motor and ball screw mechanism (Suda and Shiiba, 1996, Kawamoto et al., 2007, Li, et al., 2013) or a rack and pinion mechanism (Balarama and Rassem, 1992, Roshan, et al., 2015, Xhang, et al., 2016), have been developed, but they are different from the damper proposed in the present study. The author's group has also developed an electromagnetic damper using a ball screw mechanism, flywheel, and generator (Matsuoka, et al., 2007, 2008, 2009, Sunakoda, et al., 2009), and they confirmed its performance by applying it to a structure (Nakamura, et al., 2014). That damper had a gear to magnify and compensate for the resisting force, so the damping effect caused by the generator and the inertia effect due to rotating parts were magnified by the gear, and also response was mechanically influenced by delay on the gear. Moreover, it had a few limitations such as large size, high complexity, and energy losses of contact parts such as the gear, coupling, and motor armature. If it is possible to fabricate a damper without contact parts, it would be more efficient, compact, and sensitive. In this paper, a semiactive-type electromagnetic damper equipped with a brushless 
direct drive DC motor, which is comparatively compact by mean of simple mechanism and more efficient since no friction, was developed to solve the above problem. The damper comprises a ball screw mechanism and a brushless direct drive DC motor, which acts as a generator. This motor, which consists of two parts, a rotor with permanent magnets and a stator with coils and ferrite cores, is installed at the end of the screw shaft. Linear motion is converted into rotational motion by the ball screw mechanism, and then, the rotor is rotated. A harmonic drive voltage, that is electromotive force (EMF), is generated by electromagnetic induction, and it is proportional to the rotation speed. A damping effect is caused by energy dissipation at the resistances. EMF generates harmonic torques of each phase, but the torques are saturated in high frequency range due to limitation of the current, which makes theory of the damping effect complicated. To investigate the above, the damper was manufactured, the theories of resisting force and rise time were introduced, and performance tests were carried out. The resisting force characteristics were measured in the cases of a delta-connected winding and a star-connected winding configurations, and also the response characteristics were measured using a solid state relay (SSR). Finally, the experimental result were compared with the theoretical results, and the theories and the dynamic properties of the damper were confirmed.

\section{Specifications}

A design diagram of the electromagnetic damper developed herein is shown in Fig. 1, and its physical parameters are listed in Table 1. The damper comprises a ball screw mechanism and a brushless direct drive DC motor. The ball screw mechanism consists of a screw shaft and a ball nut, and it is supported by two angular contact ball bearings that are settled back-to-back. The brushless direct drive DC motor, Kollmorgen TBM-6013-A, consists of a rotor equipped with 12 pole permanent magnets and a stator equipped with three-phase coils and cores. The rotor is fixed at the tip of the screw shaft. The stator is installed onto the steel housing frame along with a cylindrical insulator made of plastic, and the gap between the rotor and the stator is less than $1 \mathrm{~mm}$. This type of motor has no Hall sensor because it acts as a generator. It is not necessary to install any bearing, support, and gear box, which is why the motor is so compact. When the rod end moves, its linear motion is converted into rotational motion of the screw shaft by the ball nut, and the rotor is rotated. Kinetic energy can be dissipated electrically at resistances. The electrical resistances of the three phases

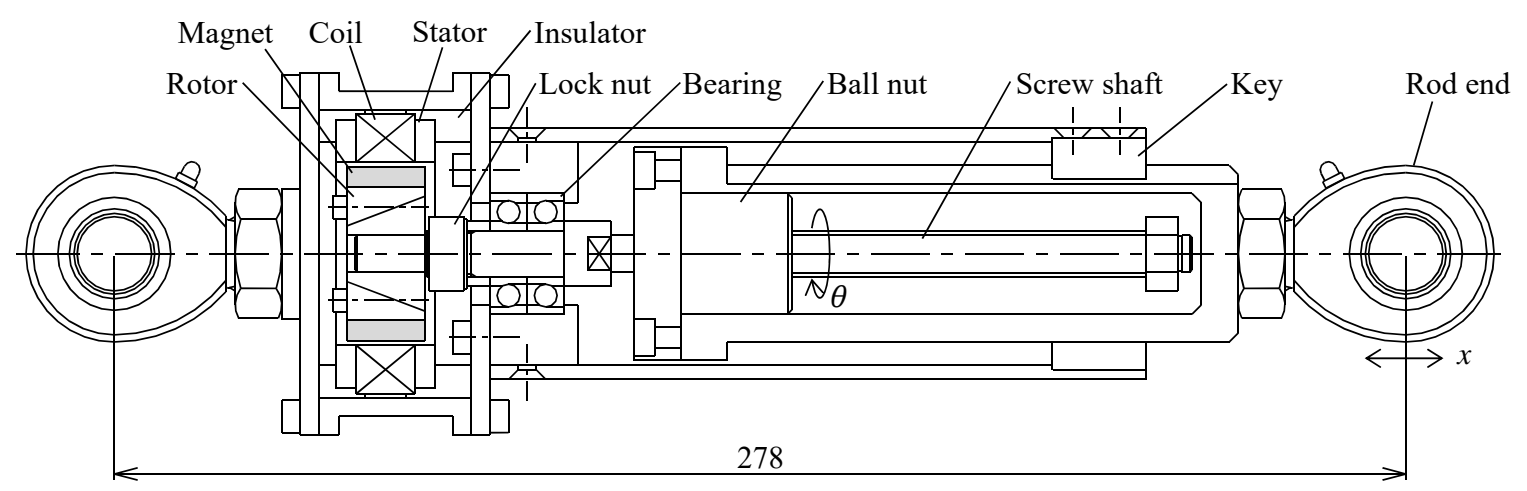

Fig. 1 Design diagram of the electromagnetic damper. A rotor is directly fixed at the tip of the screw shaft, and a stator is installed in the housing along with an insulator. There is a small gap between the rotor and the stator.

Table 1 Specification sheet.

\begin{tabular}{llll}
\hline Ball screw & Lead & $L$ & $4 \mathrm{~mm}$ \\
& Rotary efficiency & $\eta$ & 0.94 \\
& Moment of inertia & $J_{S}$ & $5.03 \times 10^{-6} \mathrm{kgm}^{2}$ \\
\hline DC motor & Torque sensitivity & $K_{T}$ & $0.087 \mathrm{Nm} / \mathrm{A}$ \\
& Motor constant & $K_{E}$ & $0.087 \mathrm{Vs} / \mathrm{rad}$ \\
& Armature resistance & $R_{a}$ & $0.804 \Omega$ \\
& Continuous current & $I_{\max }$ & $5.51 \mathrm{~A}$ \\
& Moment of inertia & $J_{m}$ & $1.41 \times 10^{-5} \mathrm{kgm}^{2}$ \\
& Inductance & $L_{m}$ & $0.39 \mathrm{mH}$ \\
\hline SSR & Operate time & & $1 \mathrm{~ms}$ \\
\hline
\end{tabular}


should be the same to avoid the generation of circulating currents in the windings inserted into each terminal of the coil according to the following two patterns. The first pattern is a delta-connected winding configuration, which is triangular, as shown in Fig. 2(a), and the second pattern is a star-connected winding configuration, which is Y-shaped, as shown in Fig. 2(b).

\section{Theory}

To investigate the basic dynamic properties of the damper, first, the characteristics of the resisting force in terms of damping torque and inertia torque are introduced theoretically. Next, the response characteristics are estimated in terms of rise time.

\subsection{Damping torque}

The instantaneous electromagnetic torque of each stator phase is caused according to the Lorentz force theorem and is expressed as a function of the EMF and the currents of each phase when the rotor is rotating (Gieras and Wing, 2002). The EMF voltage $E_{f}$ is expressed as a function of the rotation speed of the rotor, and it is given as follows:

$$
E_{f}=K_{E} \frac{d \theta}{d t}
$$

where $K_{E}$ is an EMF constant, and $\theta$ is the rotation angle of the screw shaft together with the rotor in Fig. 1. Assume that the instantaneous value of back EMF is induced in a single stator conductor by the first harmonic of the magnetic flux density. Then, the harmonic drive voltage $E_{i}$ of the $i$-th phase is given as follows:

$$
E_{i}=E_{f} \sin \left(\omega t+\phi_{i}\right)
$$

where $\omega, t$, and $\phi_{i}$ are the angular velocity of the rotor, time, and $i$-th phase angle, respectively. In this case, the motor has three phases, $i=1,2,3, \phi_{1,2,3}=-2 \pi / 3,0,2 \pi / 3$, the voltage per phase is rewritten in a matrix form as follows:

$$
\left\{\begin{array}{l}
E_{1} \\
E_{2} \\
E_{3}
\end{array}\right\}=E_{f}\left\{\sin \left(\omega t-\frac{2}{3} \pi\right) \quad \sin (\omega t) \quad \sin \left(\omega t+\frac{2}{3} \pi\right)\right\}^{T}
$$

From Kirchhoff's law, the instantaneous voltage per phase $E_{i}$ in case of the delta-connected winding configuration where one phase passes through only one resistance at the terminal, as shown in Fig. 2(a), it is given as follows:

$$
E_{i}=\sum R I_{i}+L_{m} \frac{d I_{i}}{d t}
$$

where $R$ is the total resistance of the circuit and $L_{m}$ is the synchronous inductance per phase. From Ohm's law, the instantaneous current $I_{i}$ per $i$-th phase of each circuit in case of the delta-connected winding is given as follows:

$$
I_{i}=\frac{E_{i}}{\sqrt{\left(R_{a}+R_{t}\right)^{2}+\left(\omega L_{m}\right)^{2}}} \angle \psi
$$

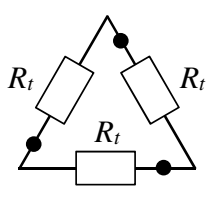

(a) Delta-connected winding

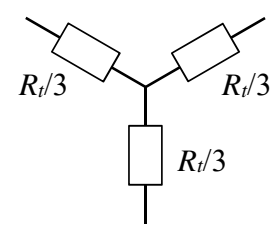

(b) Star-connected winding

Fig. 2 Winding configurations of resistance. The “•” symbols denote relays. 
where $R_{a}$ is the armature resistance per phase and $R_{t}$ is the resistance connected at the terminal. $\psi$ is a load angle which is defined as $\psi=\tan ^{-1}\left\{\omega L_{m} /\left(R_{a}+R_{t}\right)\right\}$. In case of the star-connected winding configuration, as shown in Fig. 2(b), $R_{t}$ in Eq. (5) is replaced with $R_{t} / 3$ according to the delta-Y transform.

The torque $T_{i}$ of the $i$-th phase is expressed as a function of the current, and it is given as follows:

$$
T_{i}=K_{T} I_{i} \sin \left(\omega t+\phi_{i}+\psi\right)
$$

where $K_{T}$ and $I_{i}$ are the torque constant of the motor and the instantaneous current, respectively. The torque per phase is rewritten in a matrix form as follows:

$$
\left\{\begin{array}{l}
T_{1} \\
T_{2} \\
T_{3}
\end{array}\right\}=K_{T}\left\{I_{1} \sin \left(\omega t-\frac{2}{3} \pi+\psi\right) \quad I_{2} \sin (\omega t+\psi) \quad I_{3} \sin \left(\omega t+\frac{2}{3} \pi+\psi\right)\right\}^{T}
$$

Therefore, from Eq. (7), the damping torque $T_{D}$ can be expressed as sum of all of the torques as follows:

$$
\begin{aligned}
T_{D}=\sum_{i=1}^{3} T_{i} & =K_{T}\left\{I_{1} \sin \left(\omega t-\frac{2}{3} \pi+\psi\right)+I_{2} \sin (\omega t+\psi)+I_{3} \sin \left(\omega t+\frac{2}{3} \pi+\psi\right)\right\} \\
& =K_{T}\left\{\begin{array}{lll}
I_{1} & I_{2} & I_{3}
\end{array}\right\}\left\{\sin \left(\omega t-\frac{2}{3} \pi+\psi\right) \quad \sin (\omega t+\psi) \quad \sin \left(\omega t+\frac{2}{3} \pi+\psi\right)\right\}^{T}
\end{aligned}
$$

The time history waveforms of one-cycle currents $I_{i}$ in Eq. (5) and torques $T_{i}$ in Eq. (6) per phase are shown in Fig. 3. From Fig. 3(a), the current becomes a normal harmonic waveform at low frequency and high resistance. However, from Fig. 3(b), the current is saturated with a limit, which is the continuous current $I_{\max }$ in Table 1, indicated by the red dotted lines at high frequency and low resistance, and the current becomes a flat-top square-like waveform. Therefore, the shapes of the torque are distorted slightly near each peak. If all of $I_{i}$ are within the limit, from Eqs. (1), (3) and (5), $I_{i}$ is given as follows:

$$
I_{i}=\frac{K_{E}}{\sqrt{\left(R_{a}+R_{t}\right)^{2}+\left(\omega L_{m}\right)^{2}}} \sin \left(\omega t+\phi_{i}\right) \frac{d \theta}{d t} \quad\left(I_{i}<I_{\max }\right)
$$

however, when one of $I_{i}$ is over the limit depending on the rotation speed and the resistance, $I_{i}$ is given as follows:

$$
I_{i}=I_{\max } \quad\left(I_{i} \geq I_{\max }\right)
$$

Note that the damping torque contains a scalar product of the currents and sinusoidal functions, and is linearly
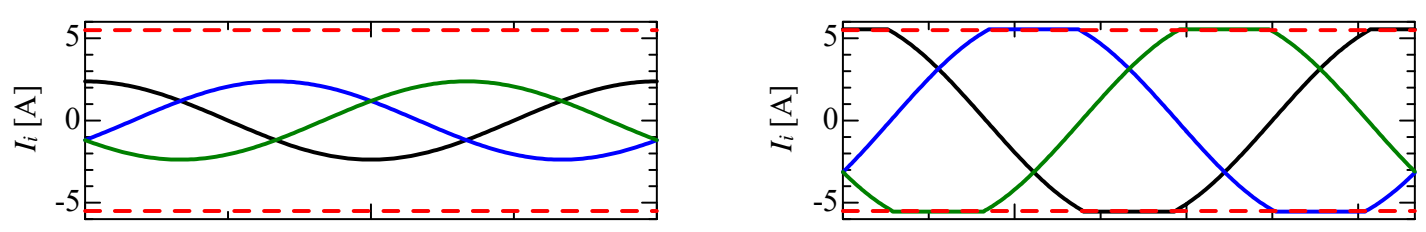

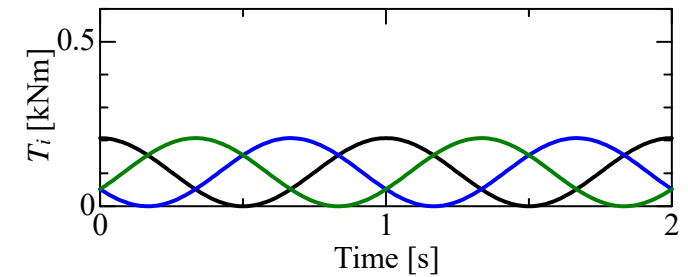

(a) Within the limit, $R_{t}=1 \Omega$, at $0.5 \mathrm{~Hz}$.

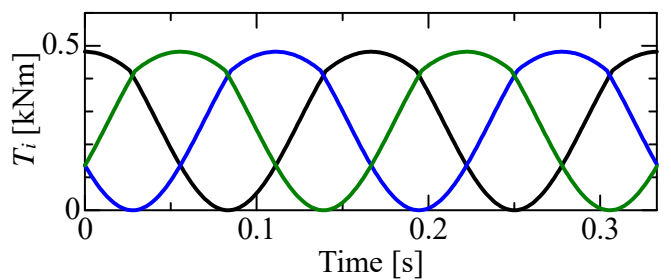

(b) Over the limit, $R_{t}=3.3 \Omega$, at $3 \mathrm{~Hz}$.

Fig. 3 Time history waveforms of currents and torques. The red dotted lines indicate the limit. The currents are normal harmonic waveforms in (a) and are saturated to the limit similar to a flat-top in (b). 
proportional to the angular velocity.

\subsection{Inertia torque}

Inertia torque $T_{I}$ is caused by the rotating parts of the rotor and the screw shaft, and it is given as follows:

$$
T_{I}=\left(J_{m}+J_{s}\right) \frac{d^{2} \theta}{d t^{2}}
$$

where $J_{m}$ and $J_{s}$ are the moments of inertia of the rotor and the screw shaft, respectively.

\subsection{Resisting force characteristics}

In general, a ball screw mechanism can efficiently convert a linear motion into a rotating motion, and the relationship between total torque $T\left(=T_{D}+T_{I}\right)$ and axial force $F$ algebraically yields the following:

$$
F=\frac{1}{\eta}\left(\frac{2 \pi}{L}\right) T
$$

where $L$ is the lead and $\eta$ is the rotary efficiency of the ball screw. The relationship between the rotating angle $\theta$ and the linear displacement of the ball nut $x$ in Fig. 1 is given as follows:

$$
\theta=\frac{2 \pi}{L} x
$$

Therefore, from Eqs. (8) - (13), the total resisting force along the axial direction, which is related to $x$, within the limit becomes

$$
F=\frac{1}{\eta}\left(\frac{2 \pi}{L}\right)^{2}\left[\frac{K_{T} K_{E}}{R_{a}+R_{t}}\left\{\sin ^{2}\left(\omega t-\frac{2}{3} \pi\right)+\sin ^{2}(\omega t)+\sin ^{2}\left(\omega t+\frac{2}{3} \pi\right)\right\} \frac{d x}{d t}+\left(J_{m}+J_{s}\right) \frac{d^{2} x}{d t^{2}}\right]
$$

and the sum of the sinusoidal functions squared in Eq. (14), which is enclosed in curly brackets, becomes 1.5 mathematically. The effect of inductance is negligibly small in particularly low frequency range, so it is assumed that $L_{m}$ is usually ignored. In case the a peak value of harmonic current per phase is always under the permitted continuous current, as shown in Fig. 3(a), Eq. (14) is simply expressed as follows:

$$
F=\frac{1}{\eta}\left(\frac{2 \pi}{L}\right)^{2}\left\{\frac{3 K_{T} K_{E}}{2\left(R_{a}+R_{t}\right)} \frac{d x}{d t}+\left(J_{m}+J_{s}\right) \frac{d^{2} x}{d t^{2}}\right\}
$$

It is clear from Eq. (15) that the damping coefficient, which is simply proportional to velocity, is constant and 1.5 times larger than conventional damping. The factor proportional to acceleration is defined by an equivalent inertia mass.

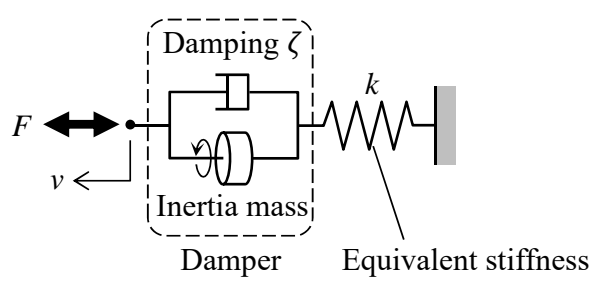

Fig. 4 Analytical model. It is assumed that a stiffness, which is a substitute for mechanical parts, is connected to the damper in series. $F$ and $v$ are the force and the velocity. 


\subsection{Response characteristics}

To investigate the rise time in the case of step input, response characteristics are determined analytically. Eq. (4) is converted using Laplace transform into an s-domain function, and the transfer function $G$ is derived as a first-order system, and as follows:

$$
G(s)=\frac{I(s)}{E(s)}=\frac{1}{R+s L_{m}}=\frac{1 / R}{1+s T_{E}}
$$

where $I(s)$ and $E(s)$ are the current and the voltage per phase in the s-domain, and $T_{E}\left(=L_{m} / R\right)$ is an electrical time constant. When a unit step function of the voltage is input, an electrical rise time $\Delta T$, which increases from 10 to $90 \%$ of the target, is given as follows:

$$
\Delta T=T_{E} \log _{e} 9
$$

Moreover, to consider elastic deformation in mechanical parts such as pins and a fixed wall, it is assumed that an analytical model has the damper, which has the damping and inertia mass of Eq. (15), and an equivalent stiffness in series, which is defined by $k$, as shown in Fig. 4 . The impedance $Z$ related to the velocity is given as follows:

$$
Z(s)=\frac{F(s)}{V(s)}=k \frac{s+2 \zeta \omega_{n}}{s^{2}+2 \zeta \omega_{n} s+\omega_{n}^{2}}
$$

where $F(s)$ and $V(s)$ are the force and the velocity of a linear motion in the s-domain, $\omega_{n}$ and $\zeta$ are the natural frequency and the damping ratio as follows:

$$
\begin{aligned}
& \omega_{n}=\frac{L}{2 \pi} \sqrt{\frac{k}{J_{m}+J_{s}}} \\
& \zeta=\frac{3 \pi K_{E} K_{T}}{\left.2 \sqrt{k\left(J_{m}+J_{s}\right)\left\{\left(R_{a}+R_{t}\right)^{2}+\left(\omega L_{m}\right)^{2}\right.}\right\}}
\end{aligned}
$$

Therefore, the transfer function of mechanical system is expressed as a second-order system. When a unit step function of the velocity is input, from Eq. (18) the force becomes

$$
\begin{aligned}
F(s)=Z(s) V(s) & =k \frac{s+2 \zeta \omega_{n}}{s^{2}+2 \zeta \omega_{n} s+\omega_{n}{ }^{2}} \frac{1}{s} \\
& =k \frac{2 \zeta}{\omega_{n}}\left\{\frac{1}{s}-\frac{s+\zeta \omega_{n}}{\left(s+\zeta \omega_{n}\right)^{2}+\omega_{n}{ }^{2}\left(1-\zeta^{2}\right)}+\frac{1-2 \zeta^{2}}{2 \zeta \sqrt{1-\zeta^{2}}} \frac{\omega_{n} \sqrt{1-\zeta^{2}}}{\left(s+\zeta \omega_{n}\right)^{2}+\omega_{n}{ }^{2}\left(1-\zeta^{2}\right)}\right\}
\end{aligned}
$$

Eq. (21) is converted by the inverse Laplace transform into a time-domain function, and the transient response is given as follows:

$$
F(t)=k \frac{2 \zeta}{\omega_{n}}\left\{1-e^{-\zeta \omega_{n} t} \cos \left(\sqrt{1-\zeta^{2}} \omega_{n} t\right)+\frac{1-2 \zeta^{2}}{2 \zeta \sqrt{1-\zeta^{2}}} e^{-\zeta \omega_{n} t} \sin \left(\sqrt{1-\zeta^{2}} \omega_{n} t\right)\right\} v
$$

where $v$ is a constant velocity such as a step function.

\section{Performance tests}

To confirm the theory mentioned above and the dynamic properties of the damper, resisting force and response 
characteristics were investigated. Performance tests were carried out using an electrical servo actuator that has a capacity of $20 \mathrm{kN}$. The test setup is shown in Fig. 5. The damper was connected to a fixed wall, which was composed of steel plates of $16 \mathrm{~mm}$ thick, and the actuator. Signals of force and displacement were input through an amplifier to an AD converter, dSPACE DS1104, and recorded by a computer.

\subsection{Resisting force characteristics}

Force and displacement were measured using a load cell and a displacement transducer when the damper was forcibly subjected to a sinusoidal wave of amplitude $10 \mathrm{~mm}$ and frequency ranging from 0.5 to $3.0 \mathrm{~Hz}$. Cement-type electrical resistances of magnitudes $1,3.3$, and $10 \Omega$ were inserted into the delta-connected winding, as shown in Fig. 2(a), and the star-connected winding, as shown in Fig. 2(b), for testing; the case without any resistance, similar to an open circuit, was tested as well. The experimental results together with the calculated results in the case of the delta-connected winding are shown in Fig. 6, and the experimental results in the case of the star-connected winding are shown in Fig. 7. It is obvious from Fig. 6 that the damper has a damping effect, which is described by elliptical forms, and an inertia effect akin to negative stiffness, which is described by the leftward rising slopes. The black chained lines indicate the theoretical inertia effect, and the equivalent inertia mass in Eq. (15) becomes $50.2 \mathrm{~kg}$. The shapes become perfectly elliptical at lower frequency and higher resistance whenever the current per phase is always less than the limit of 5.5 A in every step, as in Fig. 3(a). Otherwise, the shapes appear to be ovals with a small flat-top at higher frequency and lower resistance because the current is partially over the limit, as in Fig. 3(b). There are a little error between the

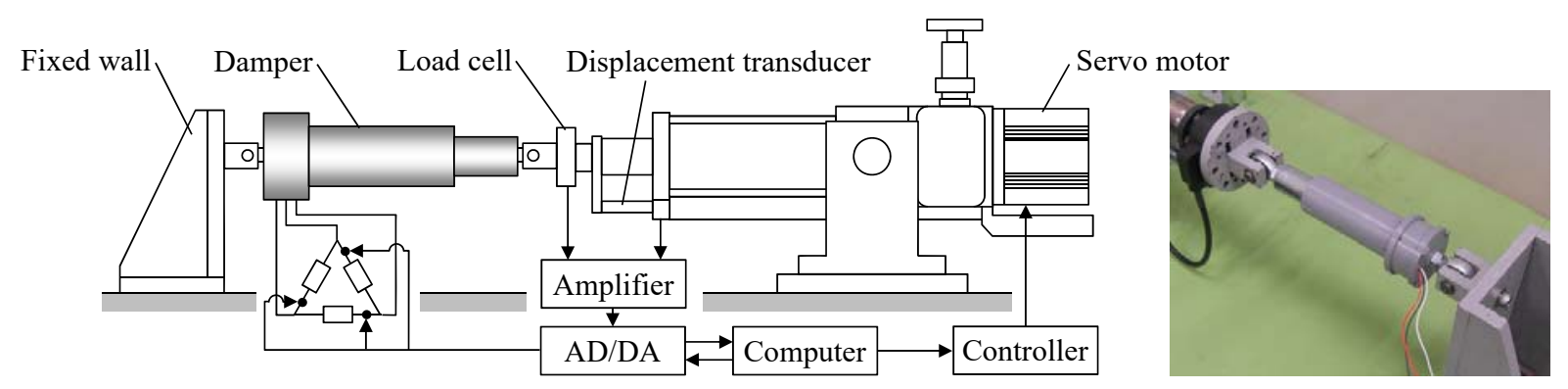

Fig. 5 Performance test setup. Both ends of the damper were connected by rod ends and pins to a fixed wall and an actuator. The damper was subjected to a sinusoidal or a triangle waves horizontally by the actuator. A servo motor was controlled by a computer through a controller. The windings were inserted into each terminal of the coil. Force and displacement were measured by the computer through an amplifier and an $\mathrm{AD}$ converter. A command signal of step function was input from the computer through a DA converter to relays in response characteristic tests.
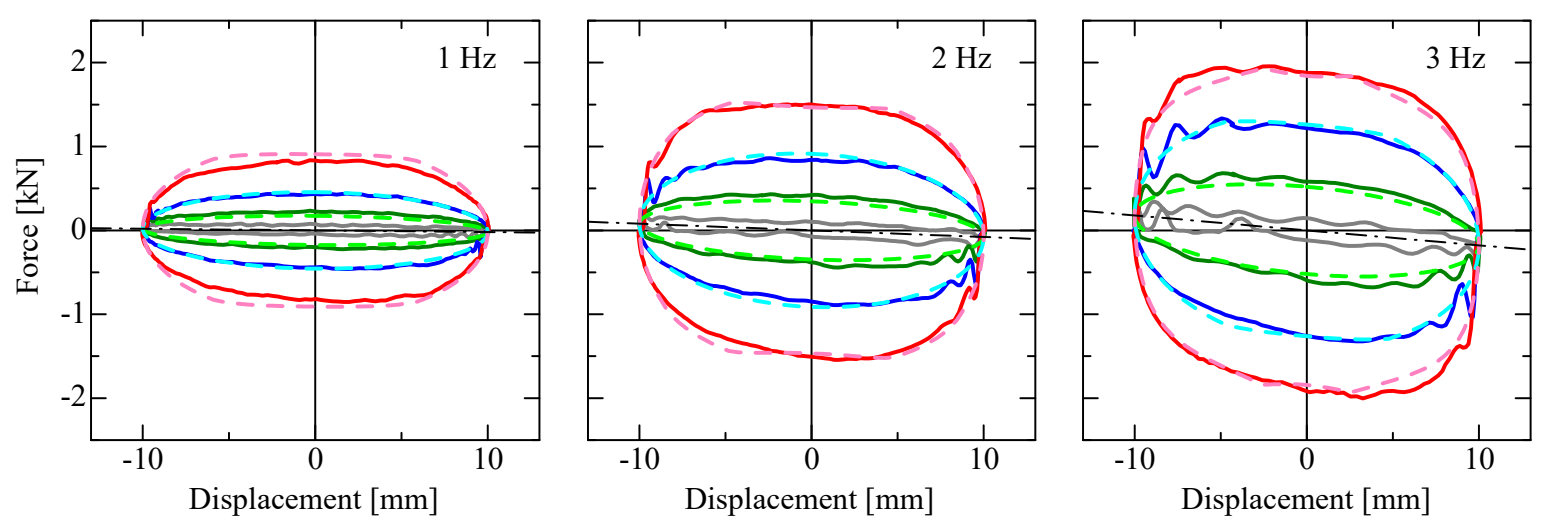

Fig. 6 Resisting force characteristics in the case of the delta-connected winding. Experiment; - open, $-10 \Omega,-3.3 \Omega,-1$ $\Omega$, Calculation; - - inertia effect, - - $10 \Omega,--3.3 \Omega,--1 \Omega$. The black chained lines indicate the theoretical inertia effect. The elliptical shapes denote the damping effect. The shapes become perfectly elliptical at low frequency and high resistance within the limit. Otherwise, they change to an oval with a flat-top at high frequency and low resistance over the current limit owing to saturation. 

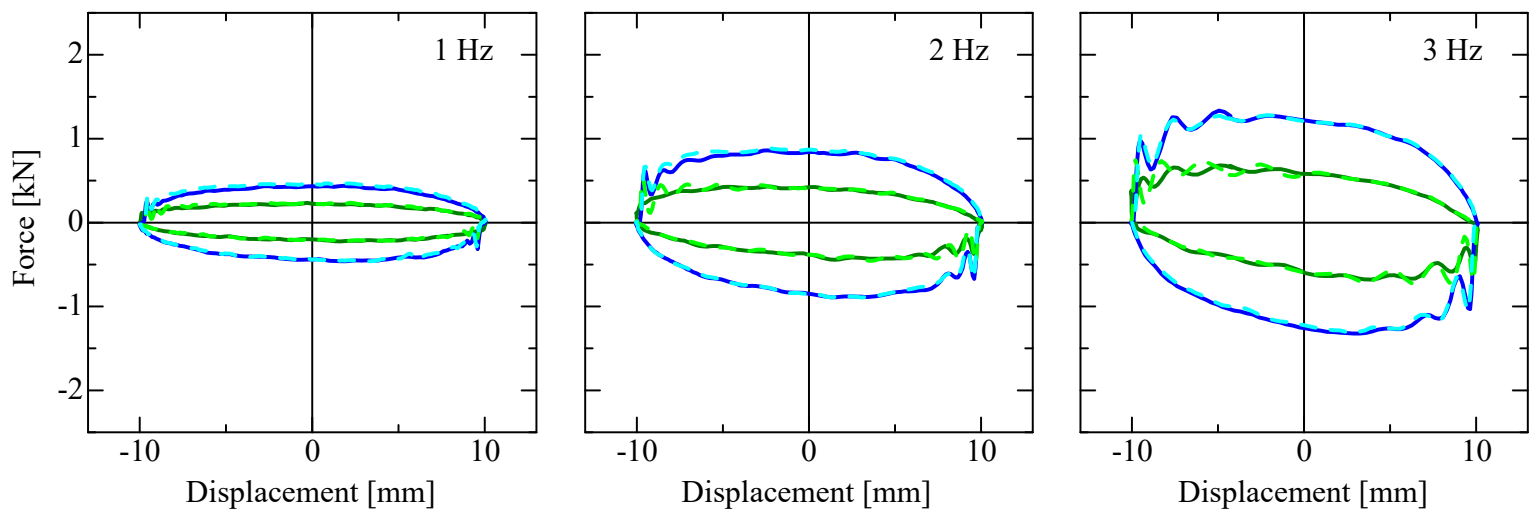

Fig. 7 Resisting force characteristics in the case of the star-connected winding. Star-connected; - - $3.3 \Omega,--1 \Omega$, Delta-connected; $-10 \Omega,-3.3 \Omega$. The results of star-connected winding are the same results as the delta-connected winding, which are shown in Fig. 6.

experimental and calculated results. It is considered that the current is probably slow curve during over the limit in fact, and not an exactly square, as shown in Fig. 3(b). It is confirmed from Fig. 7 that the experimental results in the case of the star-connected winding, when the resistance magnitudes are $1 / 3$ times to those of the delta-connected; 1 and $3.3 \Omega$, are equivalent to the results using 3.3 and $10 \Omega$ in the case of the delta-connected winding. The relationship between the damping force, which is each vertical-intercept in Fig. 6, and the velocity, which is a maximum speed during sinusoidal wave, in the case of the delta-connected winding is plotted in Fig. 8. The dotted thin lines denote the border of the current limit. From Fig. 8, the damping coefficient is linear at about 3, 6.6 and $12 \mathrm{kNs} / \mathrm{m}$ in the cases of the 10, 3.3 and $1 \Omega$ resistances in the low frequency range within the border, but it is slightly saturated in the cases of the 1 and $3.3 \Omega$ resistances in the high frequency range over the border. The experimental results show a good agreement with the calculated results. The armature resistance was measured previously, and it was $0.795 \Omega$. The friction forces were identified from each vertical-intercept in Fig. 8, which means an initial static force, and they were about $20 \mathrm{~N}$. It is considered that they are influenced by a few contact parts in the bearing, the cylinder and the key. However, they are negligibly small. The vertical-intercepts were derived by a linear approximation of experimental results within the limit. It is considered that some waveform in upper-left and lower-right portions at Figs. 6 and 7 are caused by elastic deformation in mechanical parts when the actuator moves back from peak displacement. In comparison with this kind of dampers, the damping coefficient is about 9 times larger than the previous damper which has the same size of ball screw mechanism and a typical geared DC motor (Matsuoka, et al., 2007), the force curves are comparatively smooth, and the inertia effect and the friction are relatively small. The proposed mechanism makes the damper more efficient,

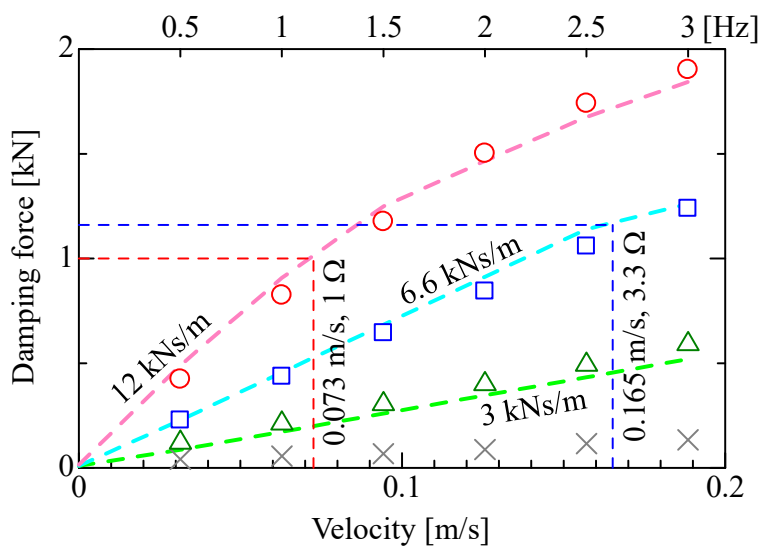

Fig. 8 Relationship between damping force and velocity in the case of the delta-connected winding. Experiment; $\times$ open, $\Delta$ $10 \Omega, \square 3.3 \Omega, \circ 1 \Omega$, Calculation; - - $10 \Omega,--3.3 \Omega,--1 \Omega$. The red and blue dotted thin lines indicate the current limits in the cases of the 1 and $3.3 \Omega$ resistances, respectively. The damping coefficients, which are initial slopes, become about 3, 6.6 and $12 \mathrm{kNs} / \mathrm{m}$. The fiction are about $20 \mathrm{~N}$. 
compact and simple, since the efficient is significantly depended on high EMF and torque constants, and the blushless direct drive has not only compact but also simple mechanism without any contact.

\subsection{Response characteristics}

The force and the command voltage were measured in a step response while the damper was forcibly subjected to a constant speed produced by a triangle wave with an amplitude of $30 \mathrm{~mm}$, frequency of $0.5 \mathrm{~Hz}$, and a sampling rate of $10 \mathrm{kHz}$ (interval $0.1 \mathrm{~ms}$ ). The command signal was input from the computer through DA converter to the electrical relays, as shown in Fig. 4, to quickly switch the circuit on and off. Solid state relays (SSR), OMRON G3TB-OA202PZ DC5-24, were installed at the black points in the delta-connected winding in Fig. 2(a) to avoid passage of the current of each phase through two SSRs in a circuit. The command voltage of about $10 \mathrm{~V}$ (9.5 V due to voltage drop) was input to the SSRs by means of a step signal at a constant speed of $60 \mathrm{~mm} / \mathrm{s}$. The resistances of $1 \Omega$ were used for testing. From Eq. (13), the rotating speed of the rotor is $94.2 \mathrm{rad} / \mathrm{s}$, and from Eq. (5), the current is $4.54 \mathrm{~A}$, which is within the limit. Therefore, the response can be calculated normally without saturation. From Eqs. (19) and (20), the natural frequency at the elastic deformation and the damping ratio become $316 \mathrm{rad} / \mathrm{s}$ and 0.38 , where $k=5.0 \times 10^{6} \mathrm{~N} / \mathrm{m}$ in Fig. 4 is adjusted by trial and error. The experimental results together with the calculated results are shown in Figs. 9 and 10. Fig. 9 is in the case of switch-on, and Fig. 10 is in the case of switch-off. From Fig. 9, it seems that the rise time, which increases from 10 to $90 \%$ between the initial and the final loads, is about $4.5 \mathrm{~ms}$. The initial load before signal input,

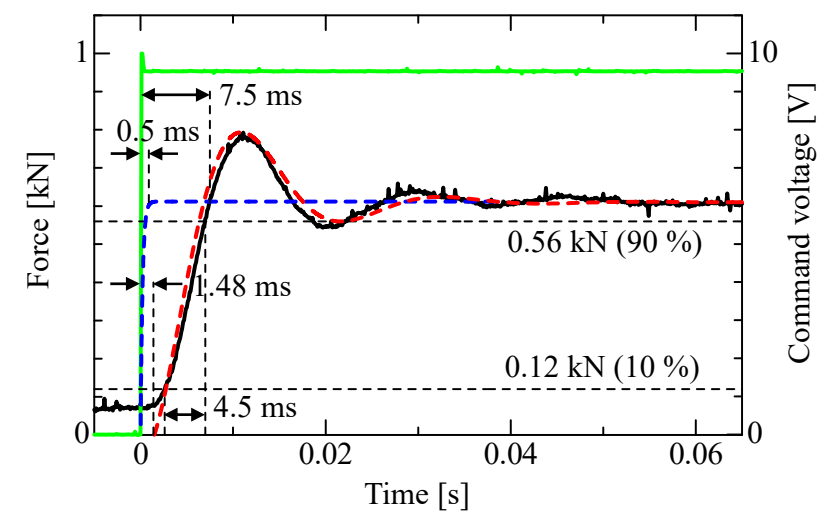

Fig. 9 Response characteristics in the case of switch-on. Experiment; - force, - command voltage, Calculation; - mechanical, - - electrical. Rise time is about $4.5 \mathrm{~ms}$, and response time becomes about $7.5 \mathrm{~ms}$. It is assumed that the time delay and the operate time of the relays make a dead time of $1.48 \mathrm{~ms}$ after signal input. The response characteristics are expressed by a second-order system.

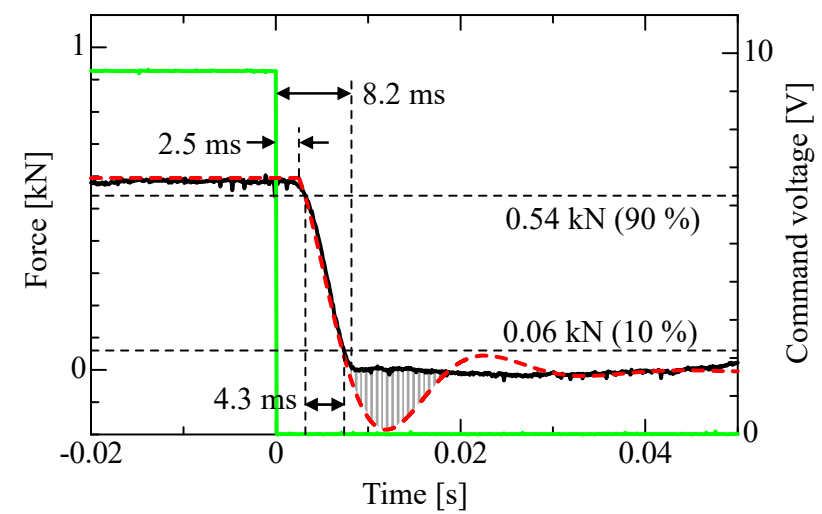

Fig. 10 Response characteristics in the case of switch-off. Experiment; - force, - command voltage, Calculation; - mechanical. Fall time is about $4.3 \mathrm{~ms}$, and response time becomes about $8.2 \mathrm{~ms}$. It is assumed that the dead time is $2.5 \mathrm{~ms}$. The response time is longer about $0.7 \mathrm{~ms}$ than that of rise. The force was not working in region of negative overshoot shaded by gray. 
which is about $60 \mathrm{~N}$, is caused by friction. The blue dotted line shows the electrical time history response waveform, which is derived by a first-order system from Eq. (16), and it takes a response time about $0.5 \mathrm{~ms}$. From Eq. (17), the electrical rise time $\Delta T$ becomes $0.475 \mathrm{~ms}$. It is considered that the operate time of the SSR, which is $1 \mathrm{~ms}$ in maximum, make a time delay electrically, and consequently the experimental results contain a small dead time at the moment. Therefore the mechanical time history response waveform as the red dotted line, which is derived by a second-order system from Eq. (22), is shifted to $+1.48 \mathrm{~ms}$ after signal input. The experimental results agree well with the calculated results. The response time of rise, which is an interval from the signal input to first across the final target, is about 7.5 ms. From Fig. 10, the fall time, which decreases from 90 to $10 \%$, is about $4.3 \mathrm{~ms}$, and is slightly shorter than the rise time. However, the response time of fall, which reach to $0 \mathrm{~N}$, is $8.2 \mathrm{~ms}$, and is longer about $0.7 \mathrm{~ms}$ than that of rise, because it is considered that the dead time is much longer about $2.5 \mathrm{~ms}$ than that of switch-on. In general, it is known that a photo coupler has a long dead time of switch-off compared to switch-on. The load after signal off in Fig. 10 is slightly different from that before signal input in Fig. 9. It is considered that release axial forces at the bearings and the ball screw. The force of negative overshooting portion, which was shaded in Fig. 9, was absorbed, and a time history force curve was converged quickly. Finally, the total response time becomes about 7.9 ms in average. In comparison with the previous damper, the response time is about 6 times faster than the previous one in the case with the geared DC motor when the damper has a gear ratio of 23.9 to generate a force of $150 \mathrm{~N}$ (Matsuoka, et al., 2007).

\section{Conclusions}

In this study, an electromagnetic damper using a brushless direct drive DC motor was developed, and theories of resisting force and response characteristics were introduced. Moreover, the dynamic properties of the proposed damper were investigated. It is reconfirmed that the damper has a damping effect and an inertia effect. The conclusions are summarized below.

1. The damping torque is caused by an EMF generated by harmonic waves, and it is given as the scalar product of harmonic currents and torques per phase.

2. From the resisting force characteristics, damping effects lead to the formation of perfect ellipses, and the effects are linear in the region of low frequency and high resistance. Otherwise, oval shapes with a flat-top are formed in the high frequency and lower resistance region owing to the current being over the limit, and the effects are nonlinear. Inertia effects and friction are relatively small.

3. From the response characteristics obtained using SSR, the rise and fall times are about $4.4 \mathrm{~ms}$ in average at a constant speed of $60 \mathrm{~mm} / \mathrm{s}$. The response time becomes around $8 \mathrm{~ms}$. The response characteristics are approximately expressed by a second-order system.

4. There was a good agreement between the experimental and calculated results, and the proposed damper theories were confirmed.

5. It is possible to make the damper more efficient, compact, and sensitive.

\section{Acknowledgement}

This work was supported by Japan Society for the Promotion of Science (JSPS) KAKENHI, Grant-in-Aid for Scientific Research (C), Grant Number JP24560278.

\section{References}

Balarama, V. M. and Rassem, R. H., Active vehicle suspension with brushless dynamoelectric actuator, US Patent No. 5091679A (1992).

Chiba, T. and Kobayashi, H., A study on damping characteristics of mechanical snubber, The thermal and nuclear power, Vol. 35, No. 3 (1984), pp. 265-273 (in Japanese).

Gieras, J. F. and Wing, M., Permanent Magnet Motor Technology, Marcel Dekker Inc. (2002), ISBN0-8247 -0739-7.

Kawamoto, Y., Suda, Y., Inoue, H. and Kondo, T., Modeling of electromagnetic damper for automobile suspension, Journal of System Design and Dynamics, Vol. 1, No. 3 (2007), pp. 524-535.

Li, Z., Zuo, L., Luhrs, G., Lin, L. and Qin, Y., Electromagnetic energy-harvesting shock absorbers: Design, modeling, 
and road tests, IEEE Transactions on Vehicular Technology, Vol. 62, No. 3 (2013), pp. 1065-1074.

Matsuoka, T., Sunakoda, K., Hiramoto, K. and Ohtake, T., Study on vibration control device using power generator, Transactions of the Japan Society of Mechanical Engineers, Series C, Vol. 73, No. 735 (2007), pp. $2926-2931$ (in Japanese).

Matsuoka, T., Sunakoda, K., Hiramoto, K. and Paul N. Roschke, Vibration tests of a three story benchmark structure with vibration control devices that generate power, 18th Analysis and Computation Specialty Conference at Structures Congress, ASCE (2008), Canada.

Matsuoka, T., Sunakoda, K., Hiramoto, K., Yamazaki, I., Fukukita, A., Paul N. Roschke, Chin-Hsiung Loh and Pei-Yang Lin, Seismic performance of vibration control device that generates power, ASME 2009 Pressure Vessels and Piping Conference, Vol. 8, No. 77695 (2009), pp. 255-262, Czech Republic.

Nakamura, Y., Fukukita, A., Tamura, K., Yamazaki, I., Matsuoka, T., Hiramoto, K. and Sunakoda, K., Seismic response control using electromagnetic inertial mass dampers, Earthquake Engineering \& Structural Dynamics, Vol. 43, No. 4 (2014), pp. 507-527.

Roshan, Y. M., Maravandi, A. and Moallem, M., Power electronics control of an energy regenerative mechatronic damper, IEEE Transactions on Industrial Electronics, Vol. 62, No. 5 (2015), pp. 3052-3060.

Sunakoda, K., Iwata, Y., Iiyama, F and Suzuki, K., Semiactive Control of Seismic Isolation System Using Mechatro Damper, Transactions of the Japan Society of Mechanical Engineers, Series C, Vol. 61, No. 584 (1995), pp. 1308-1312 (in Japanese).

Sunakoda, K., Matsuoka, T., Hiramoto, K. and Fukukita, A., Electromagnetic vibration control device applicable to twin mode of semiactive and passive types, Transactions of the Japan Society of Mechanical Engineers, Series C, Vol. 75, No. 758 (2009), pp. 2659-2664 (in Japanese).

Suda, Y. and Shiiba, T., A new hybrid suspension system with active control and energy regeneration, Journal of Vehicle System Dynamics, Supplement 25 (1996), pp. 641-654.

Xhang, Z., Zhang, X., Chen, W., Rasim, Y., Salman, W., Pan, H, Yuan, Y. and Wang, C., A high-efficiency energy regenerative shock absorber using supercapacitors for renewable energy applications in range extended electric vehicle, Applied Energy, Vol. 178 (2016), pp. 177-188. 